\title{
Elbow Joint Rehabilitation Device
}

\author{
Chai Gow Xiao, Z. Mohamad, W. R. W. Omar
}

Department of Electrical Engineering, Polytechnic Sultan Salahuddin Abdul Aziz Shah, Shah Alam, Selangor, Malaysia

Email address:

chaixiao93@gmail.com (C. G. Xiao), zunuwanas@yahoo.co.uk (Z. Mohamad), rosemehah@gmail.com (W. R. W. Omar)

\section{To cite this article:}

Chai Gow Xiao, Zunuwanas Mohamad, Wan Rosmehah Wan Omar. Elbow Joint Rehabilitation Device. International Journal of Vocational Education and Training Research. Vol. 2, No. 4, 2015, pp. 24-27. doi: 10.11648/j.ijvetr.20160204.11

Received: June 30, 2016; Accepted: July 29, 2016; Published: October 11, 2016

\begin{abstract}
Stroke is a brain disease, which it causes spasticity and may occurs muscle weakness and also joint stiffness. Besides that, joint stiffness also will occur after joint trauma and with the Continuous passive motion (CPM) exercise, the joint stiffness can be reduces and also maintain the range of motion (ROM) of the joint. Elbow Joint Rehabilitation Device provides Continuous passive motion (CPM) exercises to the patients who have elbow injury or elbow that has undergone surgical procedure and Neurological problems such as stroke and traumatic brain injuries which effected on the elbow. The purpose of this project is to upgrade the existing Elbow Joint Rehabilitation Device to more comfortable to use, more lightly in weight to be handle and carry, and also can operate in DC rechargeable batteries. To develop this project, brushed DC motor is use to move the arm/elbow joint with controllable speed and the degree of movement by using PIC microcontroller (Programmable Interface Controllers). Furthermore, a rechargeable battery is added on the device which to able the device operates in DC power supply. To conduct this project, data collection, testing and data analysis for the stroke and elbow joint injury patients. As a result, we'll find out the effectiveness of the device to the stroke and elbow injury patient.
\end{abstract}

Keywords: Stroke, Joint trauma, Joint Stiffness, Continuous Passive Motion (CPM), Elbow Joint Rehabilitation Device, PIC microcontroller

\section{Introduction}

A study of Ummi Nadiah Yusof find out that stroke is the second largest causes of Year of Life Lost in Malaysia, which ischaemic heart disease $(17.1 \%)$ is the first causes, follow with stroke $(9.6 \%)$ and the third causes was road traffic injuries $(8.3 \%)$ [1]. Stroke is a brain disease, which it causes spasticity and may occurs muscle weakness and also joint stiffness.

According the observation of the L. D. Schelosky \&J. Scott, $25 \%$ stroke patients suffer from spasticity within the first 6 weeks of the study. They have observed that spasticity primarily affects the elbow (79\% of patients), the wrist $(66 \%)$ and the ankle $(66 \%)$ [2]. Spasticity of the elbow joint will causes the arm's muscle weakness and the elbow joint stiffness.

Besides that, joint stiffness also will occur after joint trauma and with the Continuous passive motion (CPM) exercise, the joint stiffness can be reduces and also maintain the range of motion (ROM) of the joint.

A study of K. Kang, which the development of rehabilitation robot for post-stroke forearm and wrist also provided the
Continuous Passive Motion exercises for stroke patient [3]. Elbow Joint Rehabilitation Device provides Continuous passive motion (CPM) exercises to the patients who have Neurological problems such as stroke and traumatic brain injuries are a highly prevalent condition that results in high cost to the individuals and society [4]. Besides that, it is used during the first phase of rehabilitation process following an elbow injury or elbow that has undergone surgical procedure.

A rehabilitation program support the findings of Gates et al (1992) that post-operative use of CPM improves total range of motion and therefore function [5]. Thus, Continuous Passive Motion exercises are important to the patient's elbow recovery from stroke, traumatic brain injuries and also from elbow injury. By using this device, patients can do their treatment anytime and anywhere and patients can heal in a short period of time

\section{Literature Review}

\subsection{Stroke}

Stroke is a brain attack that occurs when blood flow and 
oxygen to an area of the brain is cut off. Brains control everything we do, stroke can effects an areas of the brain, which including how we move different parts of our body.

There are few symptoms of Stroke which are:

- Sudden numbness or weakness of face, arm or leg, especially on one side of the body.

- Sudden confusion, trouble speaking or understanding

- Sudden trouble seeing in one or both eyes

- Sudden trouble walking, dizziness, loss of balance or coordination

- Sudden severe headache with no known cause

The most common physical effect of stroke is muscle weakness and loss/less of control movement of arm or leg. Brain damage due to stroke can cause an arm or leg to become paralyzed and/or to develop spasticity. Spasticity refers to the abnormal symptom of having muscle are stiff and resistance in the joints when patients try to make a movement [6] [7].

\subsection{Elbow Joint}

The elbow joint is a complex hinge joint formed between the distal end of the humerus in the upper arm and the proximal ends of the ulna and radius in the forearm [8], [9]

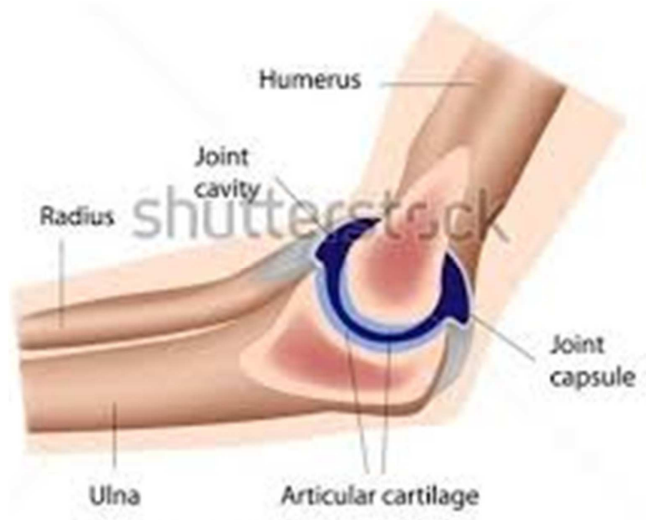

Figure 1. The structure of the elbow joint.

The movement that consists of the elbow joint are flexion and extension. The normal range of motion of the extension for elbow joint is approximate $0^{\circ}$ and the flexion of the elbow joint is approximate $140^{\circ}[8]$.

\subsection{Continuous passive motion (CPM)}

Continuous passive motion can prevent development of adhesions and contractures and thus joint stiffness [10]. Continuous passive motion is effectively to reduce the stiffness of the joint and to maintain the normal range of motion. CPM involves movement of a joint without active muscle contraction, and is accomplished with motorized devices that move the affected joint through a prescribed arc of motion for an extended period of time [11]. When the joint is in spasticity condition, increasing of the joint stiffness will be occurs. So that, continuous passive motion was apply to the hemiparesis patients can reduce the joint stiffness and maintain the range of motion of the joint. The study of Mohd Nor Azmi Ab.Patar, he had developed a robotic hand rehabilitation device, which the device provide CPM to help stroke patients reduce the joint stiffness of the finger [12].

Besides that, CPM can also help the patients who have joint injuries, which it provides a stimulating effect on the healing of tendons and ligaments, enhances healing of incisions over the moving joint, increases synovial fluid lubrication of the joint and thus increases the rate of intra-articular cartilage healing and regeneration.

\subsection{Current Technologies}

ARMin

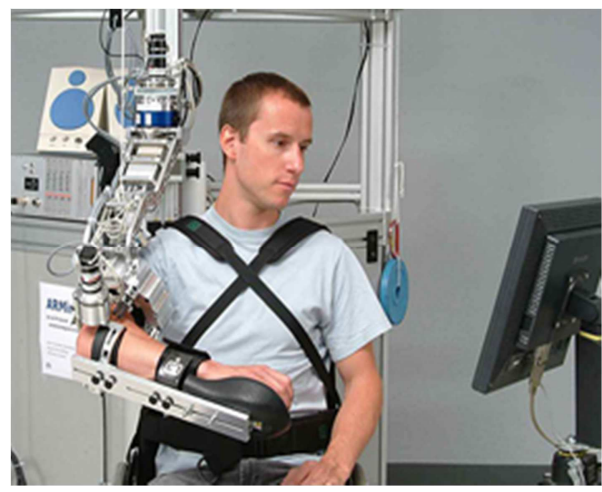

Figure 2. ARMin.

This device is used to diagnose the biomechanical changes and abnormal couplings at the shoulder, elbow and wrist joints of the impaired arm of patients post stroke [13]. This machine is bigger and unmovable. So every times the patients have to attend themselves to the hospitals for the treatment. This types of devices need AC power supply to operating. Besides that, this machine also need a widely place for the installation and it's also costly.

\subsection{Continuous Passive Motion Unit CPM (Elbow)}

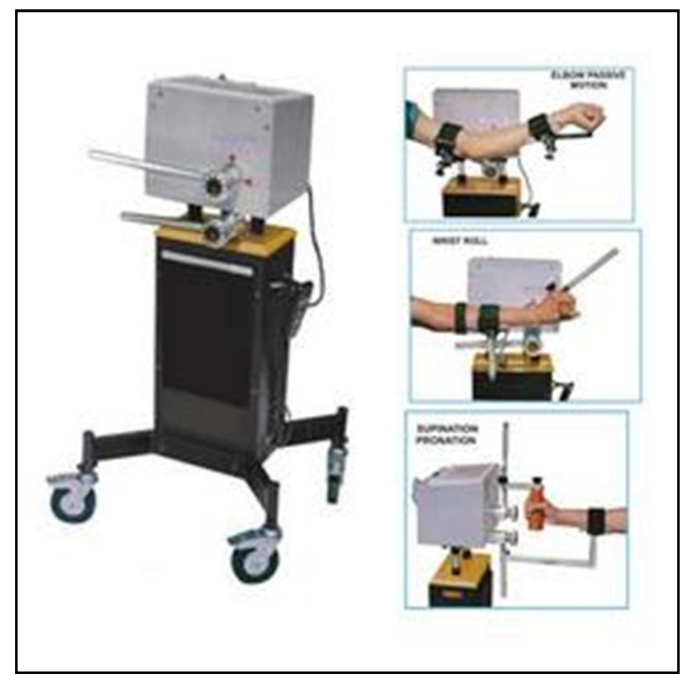

Figure 3. Continuous Passive Motion Unit CPM (Elbow).

Range of Motion (ROM) Settings for this unit are Programmable from 00 to 120deg for Elbow movements, which it can be control the range of motion for our elbow. This unit is 
attached with a stands, which it's movable, but it only limited in the area of the hospital. Besides that, this unit also need to operate by AC power supply.

\section{Methodology}

\subsection{Geared Motor}

The locomotion of the Elbow Joint Rehabilitation Device was achieved by utilizing DC geared motor. The model was use is SPG30-20k, which bought from Cytron Technologies. The rated voltage is $12 \mathrm{~V}$, with the flexible speed at $185 \mathrm{RPM}$.

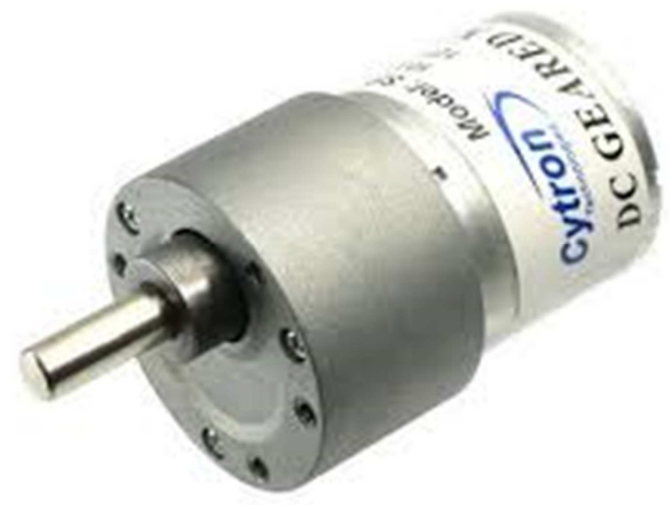

Figure. 4. DC Geared Motor SPG30-20k.

These types of motor were uses to control the upward and downward movement of the forearm. Besides that, the application of screw jack was also apply in this project, which it used as mechanical lifting. The DC geared motor was use to rotate the screw, and a nut will moves along the screw. When the motor move forward, the screw will rotate in clockwise, and the nut will moving forward to the motor, at this situation the nut is moving the forearm upward. So when the motor move reverse, the nut will moving reverse from the motor, which the nut will pull the forearm downward. The lifting nut of jack screw is used to decrease friction.

Furthermore, the angle of the device can be adjustable by changing the screw at the device that had develons. The device provided 4 difference angle around $20^{\circ}, 45^{\circ}, 60^{\circ}$ and $70^{\circ}$.

\subsection{PIC Microcontrollers}

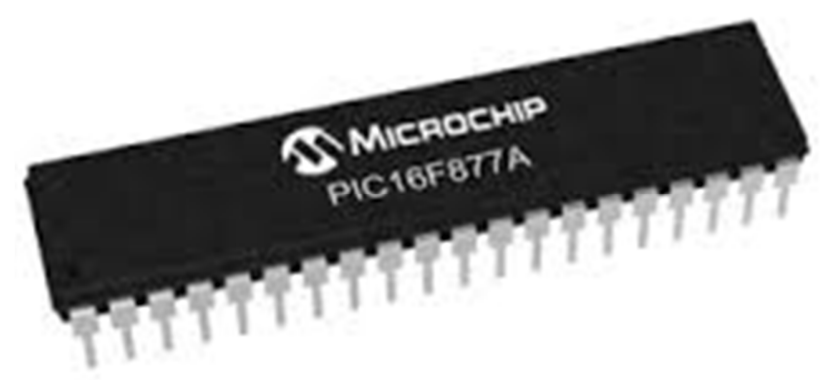

Figure 5. PIC16F877A.

PIC16F877A was decided to uses in this project, because it had 256 bytes of EEPROM Memory, which the memory that load into the PIC can be erased and rewrote easily. PIC microcontrollers act as a CPU, which control all the activities of the board and also the movement of the devices.

PIC microcontroller was uses to control the speed, count of the movement and also the level of the device. The PIC is read the setting from the user, and produces the output as the settings from the user. The user can select the speed that required, how many count needed and also the level of the angle, these are the settings that the user can decided themselves. Once the settings was done, the PIC will read the setting and produced the output depends on the requirement of the user

\subsection{Software}

Eagle 6.3.0 was used to draw the schematics diagram and create a PCB layout of the circuit. Easy Applicable Graphical Layout Editor known as EAGLE is a powerful PCB design software. This software was uses to create or produces a PCB layout of the circuit which it will be print out for the process of etching.

Besides that, MPLAB IDE v8.30 was uses as compiler of the $\mathrm{C}$ programming. MPLAB IDE supports editing, debugging and programming of Microchip 8-bit, 16-bit and 32-bit PIC microcontrollers. During this study, C language programming had used as an instruction to control the operation of the whole circuit.

C-language is chosen as the programming language for this project due to the memory size of C-language is small and easy to understand. A full program was written in $\mathrm{C}$ language and then the program will be debugged and compile by MPLAB IDE. Then by using the PIC kit 2 software to load the hex file into the PIC microcontroller.

\subsection{Data Collection}

The method that uses for data collection was distributed questionnaire. There were two test that uses for this project which is usability test and clinical test. For the usability test, data was collect in the area Electrical Engineering Department, at Polytechnic Sultan Salahuddin Abdul Aziz Shah. 30 subjects were takes for the usability test, those are the students from the Electrical Engineering Department. First, the Elbow Joint Rehabilitation Devices was used on each of the subject, difference levels was test on the subject. After the testing done, questionnaire was distributed to all the subjects.

For the Clinical test, stroke's patients and patient who have joint trauma were taken as the subject of the clinical testing. The data was collect in the hospital.

\subsection{Data Analysis}

Statistical Package for Social Sciences (SPSS) is a software package used for statistical analysis. This software was used to analysis the data of usability test and clinical test. The data was shown in a graph form by using the SPSS software.

\section{Result}

The result of the usability test, total of the subject that have 
test are 7 students which are the students from Electrical Engineering Department, at Polytechnic Sultan Salahuddin Abdul Aziz Shah. Graph below had shown that the level of the comfortability of the Elbow Joint Rehabilitation Device that have develop.

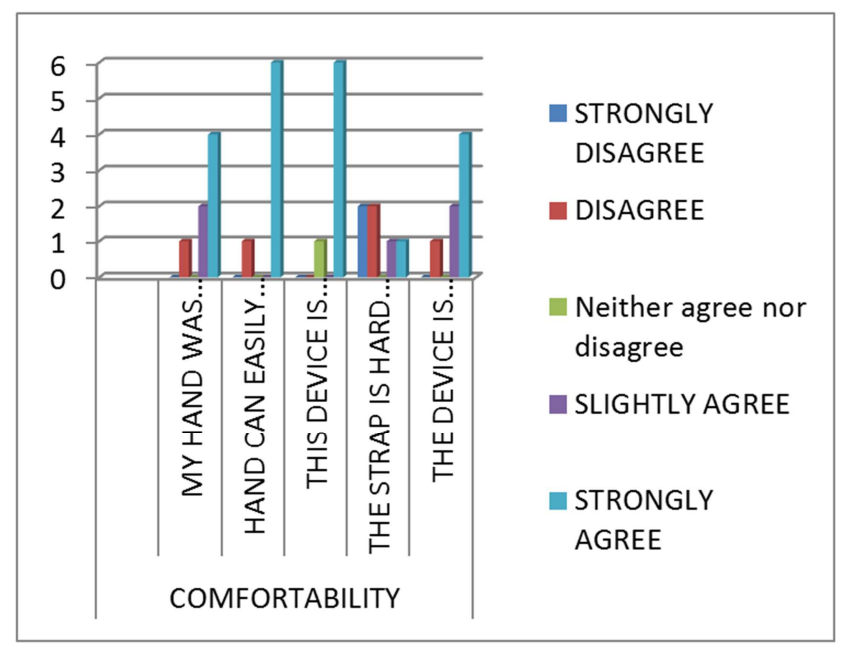

Figure 6. Comfortability of the Device.

From the graph above, 6 from the 7 students is strongly agreed that the device is easy to be use and the hands can easily fit into the device. Besides that, 4 students are strongly agreed and 2 students slightly agreed that, the placement of the hand is comfortable and also the device is less moving while operating, but only 1 of the students that disagreed with that.

\section{Conclusions}

As a conclusion, the Elbow Joint Rehabilitation Device is comfortable to be used. This study shown that the device that have develop is comfortable to be used, but there are still need some future improvement for the devices, which is the angle of the device need to be increase. The best angle should be more than $90^{\circ}$. Besides that, as a suggestion from the physiotherapist, which the count setting should be change to a timer. Because for the CPM exercise, their mostly control by time, not count of the exercise that had done.

\section{References}

[1] U. N. Yusoff, D. Mahat, A. Omar, T. C. Huey, and N. Yoep, "Burden of Premature Mortality in Malaysia," vol. 3, no. 1, 2013.

[2] L. D. Schelosky and J. Scott, "Early development of spasticity following stroke: a prospective, observational trial," pp. 1067-1072, 2010.

[3] K. Kang, P. Chin, J. Hua, H. Abdul, Y. Che, A. Leela, T. Narayanan, E. Su, and L. Ming, "Jurnal Teknologi Full paper Development of Reconfigurable Rehabilitation Robot for Post-stroke Forearm and Wrist Training," vol. 2, pp. 79-83, 2015.

[4] K. Xing, Q. Xu, J. He, Y. Wang, Z. Liu, and X. Huang, “A wearable device for repetitive hand therapy," Proc. 2nd Bienn. IEEE/RAS-EMBS Int. Conf. Biomed. Robot. Biomechatronics, BioRob 2008, pp. 919-923, 2008.

[5] E. Schuster, "Elbow Passive Motion Rehabilitation Utilizing a Continuous Passive Motion Device following Surgical Release, Manipulation Under Anesthesia, or Post Stable Fracture: A Review,” Kinex Med., p. 24, 2006.

[6] P. Rogram, "Jurnal Teknologi P ART - TASK T RAINER IN S IMULATED L EARNING,” vol. 4, pp. 71-76, 2015.

[7] S. Advocacy and A. S. R. Guide, "A Stroke Recovery Guide," 2010.

[8] F. Malagelada, B. Health, N. H. S. Trust, and J. V. Etzelclinic, Elbow Anatomy, no. JANUARY. 2014.

[9] M. T. Ghadiali and G. Surgery, "www.ghadialisurgery.com."

[10] L. A. C. Carolyn Kisner, Therapeutic Exercise Foundations and Techniques Sixth Edition. 2012.

[11] J. M. Krumberger, "Patients first!," RN, vol. 67, p. 28ac1, 2004.

[12] R. E. D. Evice, "MODEL-BASED SYSTEMS ENGINEERING OF A HAND REHABILITATION DEVICE," J. Teknol., vol. 4, pp. 101-106, 2015.

[13] L. Q. Zhang, H. S. Park, and Y. Ren, "Developing an intelligent robotic arm for stroke rehabilitation," 2007 IEEE 10th Int Conf. Rehabil. Robot. ICORR'07, vol. 00, no. c, pp. 984-993, 2007. 